has any blood or serum been effused into its substance, or into the ventricles; and what is the condition of the choroid plexus.

If the brain has not been examined in this careful manner, difficulties may arise in after stages of the inquiry, for no person can pretend to say whether it was diseased or not.

16. Examine the calvarium and the base of the skull for any fracture. -London Lancet.

\title{
HEMORRHAGE FROM THE LIVER.
}

By Edward G. Ludlow, M.D., New York.

A gentleman aged 58, of delicate frame, was taken ill on the 21 st December last. He complained of great exhaustion, with pain in the right shoulder; his pulse was 65 , and feeble; his tongue slightly coated, but moist; he was ordered pill hydg. at night, to be followed in the morning by eccoprotic mixture, with small doses of vin. tinct. colchici. Under this treatment his general symptoms improved. On the morning of the 3d January 1 saw him at 11 o'clock; be was reading the morning paper, and expressed himself better. At 1 o'clock I was sent for in great haste, and found him, on my arrival, cold and pulseless, complaining of agonizing pain in the abdomen, which was much alleviated by means of hot brandy and water, with tinct. opii and fomentations. He was relieved in about fifteen minutes, when a slight spasin of the facial muscles was observed, and he expired.

With the aid of Dr. Sabine I made a post-mortem examination, of which the following notes were taken:-

External Appearances.-Body generally pale, with slight yellowish tinge; muscles rigid.

Chest.-Lungs slightly emphysematous and studded with spurious melanotic spots.

Heart.-Normal in size and thickness; semilunar valves of left side studded with slightly ossific deposit.

Abdomen.-On opening this cavity the intestines were found floating in fluid blood and serum, which were removed with numerous coagulaamounting to several pints, "half a chamber full." On careful examination a laceration was fnund in the posterior part of the inferior concave surface of the right lobe of the liver, which was filled with a coagulum. The liver was carefully remover from the body, and on pressing the finger carefully into the mpture, it was carried into an extensive lacerated cavity extending upwards and backwards. On laying open the cavity an opening was discovered in the right hepatic vena cava, from which the fatal bemorrhage had taken place.

The rest of the viscera healthy, except the kidneys-both were encysted, particularly the left; the superior cyst of which would probably contain ten ounces.

Abercrombie, in his Pathological Researches on the Diseases of the Stomach and Liver, relates the case of a gentleman mentioned by Andral, 
previously in perfect health, who, on getting up one morning, complained of some uneasiness in the abdomen, and returned to bed, where he was left alone for some time. When his attendants returned to the room he was dead. On inspection much extravasated blood was found in the cavity of the abdomen, which appeared to have proceeded from a lacerated opening in the substance of the liver. This led to a small cavity full of coagulated blood, and the hemorrhage was distinctly traced to the rupture of a branch of the vena porta.- New York Journal of Med.

[The Editor of the New York Journal adds the following to the above.]

A case very similar to the above cane under our notice a few years since, in the person of a married lady, aged 36, the mother of several children. She rose in the morning in perfect health; and while standing before her looking-glass making her toilet, she felt a surden pain in the region of the liver; not, however, so severe as to cause any serious inconvenience at the moment. She became faint in the course of an hour, and vomited; was unable to sit up, and sent for a physician, who called me in consultation. I found her almost pulseless, with cold extremities and surface ; sunken, anxious expression of features, and a distressing sensation of sinking at the epigastrium. She grew gradually weaker and weaker, and expired in about ten hours from the time of the attack. Post-mortem examination revealed a laceration in the liver. The cavity of the abdomen contained more than a gallon of extravasated blood. All the other organs were healthy. Although no opening was found in the vena cava there can be no doubt, we think, that the hemorrhage proceeded from that vessel.

\section{CASES, BY AN OLD PHYSICIAN.}

To the Editor of the Boston Medical and Surgical Journal.

Dear Sir,-I have recently received several interesting medical communications from my veverable friend, Dr. Oliver Partridge, of Stockbridge, now in the 96th year of his age, and still enjoying a comfortable state of health. They are very curious and instructive. The doctor bas been in practice more than 67 years, and in that time he says "it has been my lot, 1st, to invent a speedy and safe remedy for breeding sickness, even if the stomach ejects all food and medicine and the abdominal spasms are seemingly intolerable. 2nd, two cases of females in constant spasms, unless in an horizontal posture or on their backs, and one given over to die as incurable by two of my superiors, after their close attendance fifteen months, tucked on to my care for leanness and debility exceeding all, like a skeleton covered with a wet cloth. 3d, I have found a new method (to me) of curing the piles, by practising on myself. 4th, I think I have much the best way of relieving a prolapsus uteri; but I find every one has the best way. 5th, in two cases, one of an arm, the other of a leg I aralyzed by mercury, I have afforded relief. I began after twenty years 